\title{
Bifurcation topology transfer in nonlinear nanocantilever arrays subject to parametric and internal resonances
}

\author{
Saoussen Souayeh ${ }^{1}$ and Najib Kacem ${ }^{1, a}$ \\ ${ }^{1}$ FEMTO-ST Institute - UMR CNRS 6174 24, chemin de l’Épitaphe, F-25000 Besançon, France
}

\begin{abstract}
The collective nonlinear dynamics of a coupled array of nanocantilevers is investigated while taking into account the main sources of nonlinearities. The amplitude and phase equations of this device, subject to parametric and internal resonances, are analytically derived by means of a multi-modal Galerkin discretization coupled with a multiscale analysis. Based on the steady-state solutions of these equations, the frequency responses are numerically computed for a two-beam array. The effects of different parameters are investigated and several dynamical aspects are confirmed by numerical simulations. Particularly, we have demonstrated that the bifurcation topology transfer is imposed by the first nanocantilever and it can be general to the collective nonlinear dynamics of the NEMS array.
\end{abstract}

\section{Introduction}

Extensive researches have been conducted, for several years in order to investigate the microelectromechanical systems (MEMS). Indeed, they are widely used in different applications such as biotechnology, automotive, aerospace and biomedicine. Following the trend of very large scale integration (VLSI), new devices called nanoelectromechanical systems (NEMS) come as a result of the size reduction of MEMS from micro down to nanometers. A NEMS is a nano-scale device composed by an electronic circuitry and a mechanical moving part allowing to detect a particular physical quantity and to convert it into a measurable electrical signal.

The principal developed nanoelectromechanical systems include nanowires, carbon nanotubes, silicon nano-beams and nanocantilevers [1]. The latter can be integrated into smart systems for control and monitoring like ultrasensitive mass detectors [2]. A NEMS oscillator has a multitude of nonlinear properties such as periodic attractors [3], bistability [4], complex dynamics [5] and bifurcation topology [6]. It has attracted considerable interest in recent years [7,8]. Consequently, arrays of coupled NEMS are modeled in order to investigate these fundamental aspects of complex nonlinear phenomena [9-11].

In addition, this type of systems is able to display internal resonances (IRs) [12] which occur between each adjacent oscillators. One of the first researchers who investigated IRs was Sethna [13]. Currently, more and more designers studied IRs in their models due to their important properties of suppressing oscillations in cantilever [12] or enhancing the coupling effect in dynamical systems [14]. In particular, several researchers have modeled arrays subject to parametric excitation. For example, Buks and Roukes [15] studied the mechanical properties of an electrically actuated array of doubly-clamped beams parametrically excited at primary resonance, Lifshitz and Cross [16] derived the equations of motion of an array composed by nonlinear

${ }^{a}$ e-mail: najib.kacem@femto-st.fr oscillators under parametric resonance and Gutschmidt et al. [17] have studied the collective dynamics of small and large arrays of NEMS subject to both parametric and internal resonances.

In this paper, the nonlinear dynamics of an array of coupled NEMS subject to parametric and internal resonances is investigated. The developed model is multiphysics and includes the main sources of nonlinearities with an expansion of the electrostatic force in Taylor series up to the fifth-order to allow the capture of the mixed behavior [1]. A perturbation technique (multiple scales method) and the Galerkin discretization method are used to transform the continuum model into a system having finite degrees of freedom. 1:1 internal resonance is considered to derive general equations of phase and amplitude modulations for each cantilever. These equations are solved numerically in order to investigate the effects of several design parameters on the collective dynamics of the considered device in terms of energy and bifurcation topology transfers.

\section{Design and Model}

The considered device is particularly composed by $N$ coupled NEMS. The first beam has a length $l_{1}$ and the $N-1$ last beams have the same length different from the first one. The first nanocantilever is subject to electrostatic forces provided by electrode $E$ as shown in Figure 1. In practice, the electrode $E$ has the same length as the first nanocantilever $\left(l_{1}\right)$, in order to actuate only the first beam, and it is subject to the electric load $v(\tilde{t})=V_{d c}+V_{a c} \cos (\tilde{\Omega} \tilde{t})$, where $V_{d c}$ is the $d c$ polarization voltage, $V_{a c}$ is the amplitude of the applied $a c$ voltage, $\tilde{t}$ is the time and $\tilde{\Omega}$ is the excitation frequency. The overhang is polarized by the $d c$ voltage allowing a linear coupling between the nanocantilevers.

\section{Analytical solving}

By means of the extension of the nonlinear equations of motion describing the flexural vibrations of a single nanocan- 


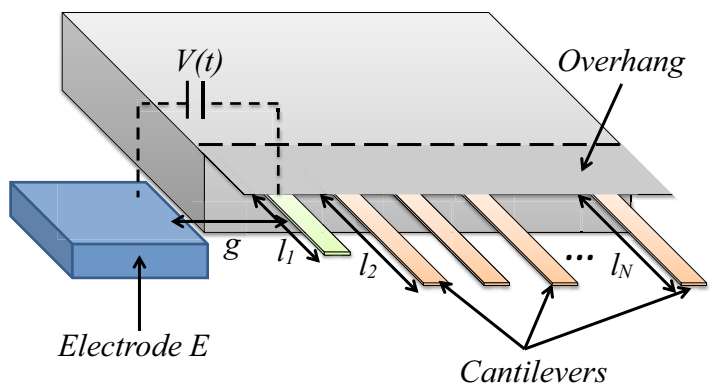

Fig. 1. An array of $N$ NEMS linearly coupled via the overhang. Only the first nanocantilever is actuated in-plane by the electrode $E$ and $l_{n}=R_{n} * l_{1}$ where $n \in\{2,4, \ldots, N\}$.

tilever electrostatically actuated and based on the extended Hamilton principle [18], the mathematical model of the design of Figure 1 is derived (Equation (1)), by taking into account the linear coupling between each adjacent nanocantilevers.

$$
\begin{aligned}
& E I \partial_{s, s, s, s} \tilde{w}_{n}+\rho b h \partial_{\tilde{t}, \tilde{t}} \tilde{w}_{n}+\frac{1}{2} \tilde{d}\left(\tilde{w}_{n+1}-2 \tilde{w}_{n}+\tilde{w}_{n-1}\right) \\
& +\tilde{c}_{n} \partial_{\tilde{t}} \tilde{w}_{n}+E I \partial_{s}\left(\partial_{s} \tilde{w}_{n} \partial_{s}\left(\partial_{s} \tilde{w}_{n} \partial_{s, s} \tilde{w}_{n}\right)\right) \\
& +\rho b h \partial_{s}\left(\partial_{s} \tilde{w}_{n} \int_{l_{n}}^{s} \partial_{\tilde{t}, \tilde{t}}\left(\int_{0}^{s_{1}}\left(\partial_{s} \tilde{w}_{n}\right)^{2} d s_{2}\right) d s_{1}\right) \\
& +\Delta_{1 n} \tilde{\eta}_{n} \tilde{c}_{n}\left(\tilde{w}_{n}^{2} \partial_{\tilde{t}} \tilde{w}_{n}\right)-\frac{1}{2} \Delta_{1 n} b \epsilon\left(\frac{\left(V_{d c}+V_{a c} \cos (\tilde{\Omega} \tilde{t})\right)}{g-\tilde{w}_{n}}\right)^{2}=0
\end{aligned}
$$

where $\partial_{s}$ and $\partial_{\tilde{t}}$ denote respectively the partial differentiation with respect to the arclength $s$ and to the time $\tilde{t}, n \in$ $\{1, \ldots, N\}, \tilde{w}_{n}(s, \tilde{t}), l_{n}, b$ and $h$ are respectively the in-plane bending deflection, the length, the width and the thickness in the direction of vibration of a nanocantilever, $\tilde{w}_{0}=$ $\tilde{w}_{N+1}=0, E$ is the Young's modulus and $I$ is the geometrical moment of inertia of the cross section, $g$ is the capacitor gap, $\rho$ is the material density, $\epsilon$ is the dielectric constant of the gap medium, $\tilde{d}$ is the linear term of coupling between each adjacent NEMS. $\Delta_{1 n}$ is the Kronecker symbol $\left(\Delta_{1 n}=1\right.$ if $n=1$ and otherwise $\left.\Delta_{1 n}=0\right)$ which indicates that only the first NEMS is subject to the electrostatic force and to the Van Der Pol damping via the parameter $\tilde{\eta}_{n}$. The boundary conditions are:

$$
\tilde{w}_{n}(0, \tilde{t})=\partial_{s} \tilde{w}_{n}(0, \tilde{t})=\partial_{s, s} \tilde{w}_{n}\left(l_{n}, \tilde{t}\right)=\partial_{s, s, s} \tilde{w}_{n}\left(l_{n}, \tilde{t}\right)=0(2)
$$

For convenience, we normalize Equations (1) and (2) by introducing the following nondimensional variables: $w_{n}=$ $\frac{\tilde{w}_{n}}{g}, x=\frac{s}{l_{1}}, t=\frac{\tilde{t}}{\tau}$, where $\tau=\frac{2 l_{1}^{2}}{h} \sqrt{\frac{3 \rho}{E}}$. We obtain the following normalized equation

$$
\begin{aligned}
& \partial_{x, x, x, x} w_{n}+\partial_{t, t} w_{n}+\frac{1}{2} d\left(w_{n+1}-2 w_{n}+w_{n-1}\right) \\
& +c_{n} \partial_{t} w_{n}+\delta_{1} \partial_{x}\left(\partial_{x} w_{n} \partial_{x}\left(\partial_{x} w_{n} \partial_{x, x} w_{n}\right)\right) \\
& +\delta_{2} \partial_{x}\left(\partial_{x} w_{n} \int_{R_{n}}^{x} \partial_{t, t}\left(\int_{0}^{x}\left(\partial_{x} w_{n}\right)^{2} d x\right) d x\right) \\
& +\Delta_{1 n} \eta_{n} c_{n}\left(w_{n}^{2} \partial_{t} w_{n}\right)-\Delta_{1 n} \delta_{3} \frac{V_{d c}}{V_{a c}}\left(\frac{1+\frac{V_{a c}}{V_{d c}} \cos (\Omega t)}{1-w_{n}}\right)^{2}=0 \\
& w_{n}(0, t)=\partial_{x} w_{n}(0, t)=\partial_{x, x} w_{n}\left(R_{n}, t\right)=\partial_{x, x, x} w_{n}\left(R_{n}, t\right)=0
\end{aligned}
$$

The expressions of the nondimensional parameters $c_{n}, \delta_{1}$, $\delta_{2}, \delta_{3}, R_{n}, \Omega, d$ and $\eta_{n}$ introduced in Equation (3) are

$$
\begin{aligned}
& c_{n}=\frac{\tilde{c}_{n} l_{1}^{4}}{E I \tau}, \delta_{1}=\left(\frac{g}{l_{1}}\right)^{2}, \delta_{2}=\frac{1}{2}\left(\frac{g}{l_{1}}\right)^{2}, \delta_{3}=\frac{6 V_{a c} V_{d c} \epsilon l_{1}^{4}}{E h^{3} g^{3}}, \\
& R_{n}=\frac{l_{n}}{l_{1}}, \Omega=\frac{\tilde{\Omega}}{\tau}, d=\frac{\tilde{d} l_{1}^{4}}{E I}, \eta_{n}=\tilde{\eta}_{n} g^{2}
\end{aligned}
$$

The ultimate goal is to derive the amplitude and phase equations of this array of NEMS. The first step is to investigate the 1:1 internal resonance in this system. The expressions of the natural frequencies $\omega_{k}$ of the NEMS corresponding to the first bending mode are obtained by using the Galerkin decomposition on Equation (3). Then, the condition of commensurability between $\omega_{k}$ is obtained by $\sum_{k=1}^{m} C_{k} \omega_{k}=0$ where $C_{k}$ are positive or negative integers and $m \geqslant 2$ is the number of the used nanocantilevers. In our case of the 1:1 IR, the integers $C_{k}$ are equal to 1 . Since only the first NEMS is electrostatically actuated, the occurence of IR between the two first beams depends on the $d c$ polarization. Furthermore, for slight linear coupling (small values of $d$ ), the closed-form solution of the driving voltage $V_{d c(1: 1)}$ which enables the existence of the 1:1 IR is identified in term of design parameters

$$
V_{d c(1: 1)}=f\left(g, h, E, l_{1}, \rho, \epsilon, R_{2}\right)
$$

Then, the modal decomposition coupled with the multiple scales method are applied on the set of the resulting equations, by considering that the first NEMS is subject to the parametric electrostatic actuation while 1:1 IR is taken into account between each adjacent NEMS. In order to describe these two conditions, we introduce the detuning parameter $\sigma$ as following

$$
\begin{aligned}
& \Omega=\omega_{1}+\xi^{2} \sigma \\
& \omega_{n+1}=\omega_{n}+\xi^{2} \sigma, n \in\{1, \ldots, N-1\}
\end{aligned}
$$

where $\xi$ is a small expansion parameter and $\omega_{n}$ is the natural frequency of the $n^{\text {th }}$ beam at the first bending mode. Yet, we derive the amplitude and phase equations corresponding to the different nanobeams.

\section{Numerical simulations}

The amplitude and phase equations are numerically solved in order to provide the frequency responses of the 1:1 IR configuration by considering the closed-form solution of the driving voltage (Equation (5)). For example, we consider the design of Table 1 to investigate the effects of the parameters $V_{a c}, \eta_{1}, Q_{1}$ and $d$ on the response of a twobeam array. These simulations are represented in Figures $2,3,4$ and 5 where $w_{1-\max }$ and $w_{2-\max }$ correspond to the maximum amplitudes respectively of the first and the second nanocantilevers, $B_{i}, i \in\{1, \ldots, 5\}$ are the bifurcation points and the continous and dashed lines are respectively the stable and unstable branches. We suppose that the two cantilevers vibrate in phase quadrature and we represent only the non-zero solutions, since they contain the main dynamical aspects. For convenience, we introduce the variable $\hat{\sigma}=\frac{\sigma}{\sigma\left(B_{3}\right)}$ which permits to display several curves on the same graph while varying design parameters, where $B_{3}$ corresponds to the peak amplitude (as shown in Figures 2, 3, 4 and 5).

\subsection{Effects of the amplitude voltage $V_{a c}$}

Firstly, the effects of $V_{a c}$ on the array's behavior are shown in Figure 2, by varying the value of the $a c$ voltage $(0.6$ and $0.9 \mathrm{~V}$ ). When increasing $V_{a c}$, the initially mixed response of the first nanocantilever of Figures 2 (a) leans to 
Table 1. Design parameters of the investigated resonator.

\begin{tabular}{lllll}
\hline Design & $l_{1}(\mu m)$ & $\mathrm{h}(\mathrm{nm})$ & $\mathrm{b}(\mathrm{nm})$ & $g(\mu m)$ \\
\hline 1 & 3 & 100 & 200 & 3.5 \\
\hline
\end{tabular}

(a)

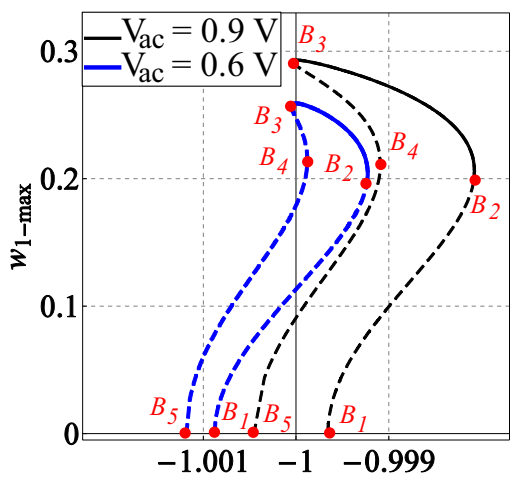

(b)

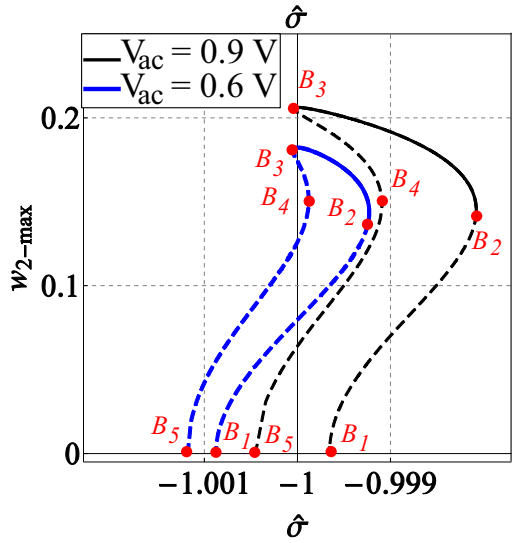

Fig. 2. Analytical forced frequency responses under 1:1 IR of (a) the first and (b) the second nanocantilevers with $l_{1}=3 \mu \mathrm{m}$, $h=100 \mathrm{~nm}, d=0.001, R_{2}=1.1, b=200 \mathrm{~nm}, Q_{1}=10000$, $\eta_{1}=300, g=3.5 \mu \mathrm{m}$ and various $V_{a c}$. The continous and dashed lines are the stable and unstable branches, respectively.

the left enabling the capture of a more pronounced mixed behavior. It is due to the fact that the equation of motion of the first beam contains the nonlinear electrostatic terms derived from the Taylor series expansion of the actuation force up to the fifth-order. Interestingly, Figure 2 (b) shows that the second nanocantilever exhibits a mixed behavior, despite the fact that its equation of motion does not contain the nonlinear electrostatic terms since it is not directly excited by the electrostatic force. This illustrates the property of the bifurcation topology transfer between the NEMS imposed by the first nanocantilever.

\subsection{Effects of the Van Der Pol parameter $\eta_{1}$}

The effects of the nonlinear parameter $\eta_{1}$ are illustrated in Figure 3. This parameter acts on the VDP damping and affects both the energy of the first beam and the energy transferred to the second NEMS. While taking into account the highest values of damping in our configurations (Figure $\left.3, \eta_{1}=700\right)$, the energy dissipation is considerable. So, the transferred energy is reduced producing small oscillation amplitudes. However, for the lowest values of damping $\left(\eta_{1}=300\right)$, the first oscillator provides higher energy (a)

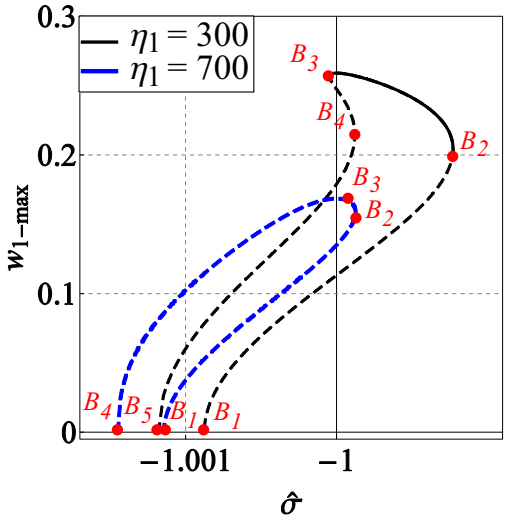

(b)

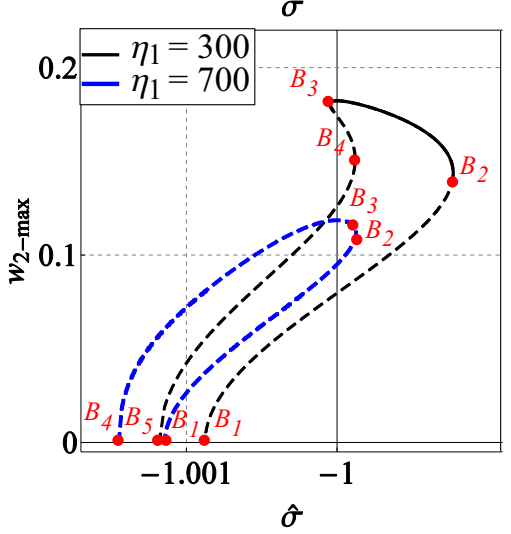

Fig. 3. Analytical forced frequency responses under 1:1 IR of (a) the first and (b) the second nanocantilevers with $l_{1}=3 \mu \mathrm{m}$, $h=100 \mathrm{~nm}, d=0.001, R_{2}=1.1, b=200 \mathrm{~nm}, Q_{1}=10000$, $V_{a c}=0.6 V, g=3.5 \mu \mathrm{m}$ and various $\eta_{1}$.

to actuate the second beam and consequently will amplify its oscillation amplitude.

\subsection{Effects of the quality factor $Q_{1}$}

The frequency responses for various values of the quality factor $Q_{1}$, are represented in Figure 4 . The quality factor parameter affects the linear dissipation mechanism via the damping parameter of the first NEMS $c_{1}$. Hence, it acts also on the Van Der Pol damping proportional to $\eta_{1} c_{1}$. If $Q_{1}$ increases, the damping parameter $c_{1}$ is reduced yielding to the decrease of the energy losses. Figures 4 (a) and (b) show a mixed-type behavior for both beams. For each nanocantilever, the modal amplitudes corresponding to $Q_{1}=$ 12000 are higher than those obtained for $Q_{1}=10000$.

\subsection{Effects of the linear coupling $d$}

Finally, we vary the value of the linear coupling $d$ while keeping the other design parameters constant. Figure 5 shows that when increasing $d(d=0.001)$, the transverse displacement amplitudes $w_{1-\max }$ and $w_{2-\max }$ are amplified. The variation of $d$ produces also the translation of the frequency response along the $\mathrm{x}$-axis. This effect is not readable in this representation because we use $\hat{\sigma}$ instead of $\sigma$.

\section{Conclusion}

We have developed an analytical model for the nonlinear dynamics of an array of $N$ coupled NEMS excited by an 
(a)

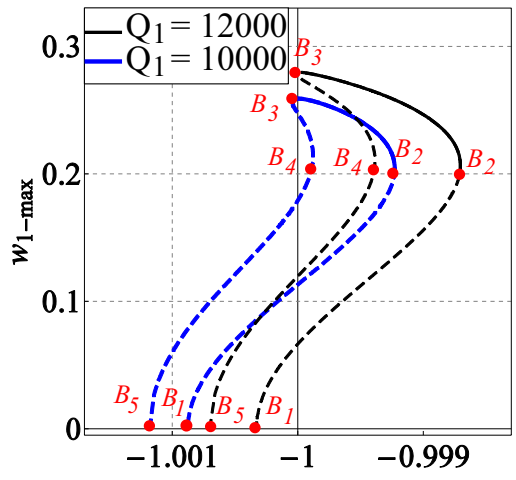

(b)

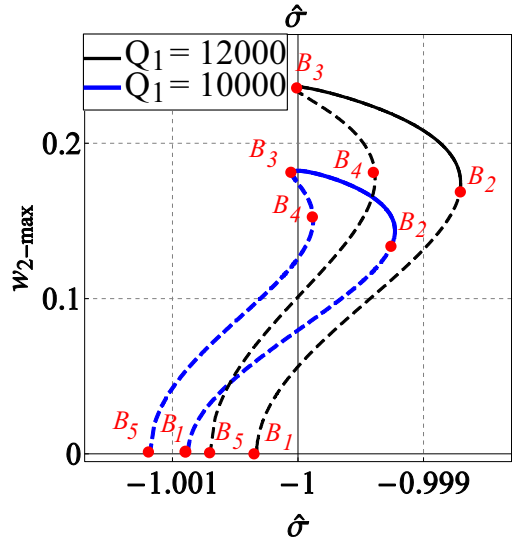

Fig. 4. Analytical forced frequency responses under 1:1 IR of (a) the first and (b) the second nanocantilevers with $l_{1}=3 \mu \mathrm{m}$, $h=100 \mathrm{~nm}, d=0.001, R_{2}=1.1, b=200 \mathrm{~nm}, V_{a c}=0.6 \mathrm{~V}$, $g=3.5 \mu \mathrm{m}, \eta_{1}=300$ and various $Q_{1}$.

(a)

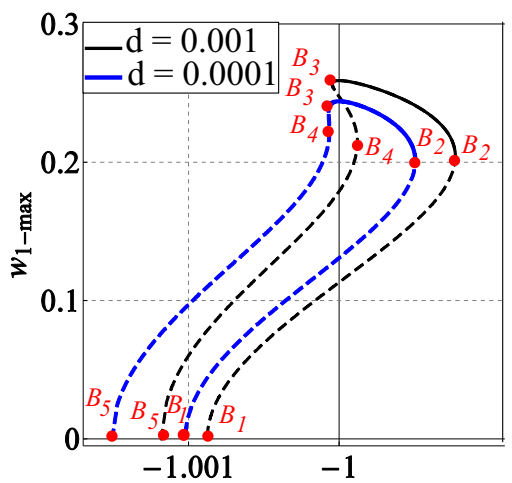

(b)

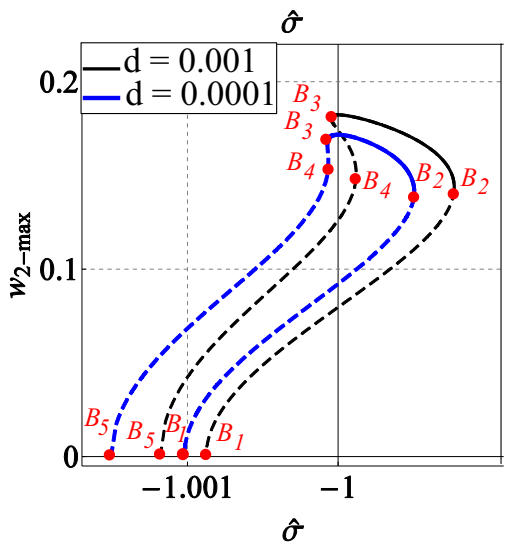

Fig. 5. Analytical forced frequency responses under 1:1 IR of (a) the first and (b) the second nanocantilevers with $l_{1}=3 \mu \mathrm{m}$, $h=100 \mathrm{~nm}, Q_{1}=10000, R_{2}=1.1, b=200 \mathrm{~nm}, V_{a c}=0.6 \mathrm{~V}$, $g=3.5 \mu m, \eta_{1}=300$ and various $d$. electrostatic force under parametric actuation and 1:1 internal resonance. This model includes electrostatic, inertial and geometric nonlinearities. In addition, the nanocantilevers are linearly coupled via the overhang.

After the investigation of internal resonance occurrence between each adjacent NEMS, the amplitude and phase equations are derived using the multiple time scales and the Galerkin discretization methods. Then, we have computed parametrically the periodic solutions of these two equations subject to 1:1 IR, with respect to the phase. Based on numerical simulations of the frequency responses, we have demonstrated the effects of several design parameters on the nonlinear behavior of a two-beam array for several configurations. Especially, we have demonstrated that the bifurcation topology is imposed by the first nanocantilever, electrostatically actuated.

\section{References}

1. N. Kacem, J. Arcamone, F. Perez-Murano, S. Hentz, Journal of Micromechanics and Microengineering 20, (2010) 045023

2. K. Jensen, K. Kim, A. Zettl, Nature Nanotechnology 3, (2008) 533-537

3. I. Kozinsky, H. W. Ch. Postma, O. Kogan, A. Husain, M. L. Roukes, Physical Review Letters 99, (2007) 1-5

4. N. Kacem, S. Hentz, D. Pinto, B. Reig, V. Nguyen, Nanotechnology 20, (2009) 275501

5. X. Ni, L. Ying, Y.-C. Lai, Y. Do, C. Grebogi, Physical Review E 87, (2013) 052911

6. N. Kacem, S. Hentz, Applied Physics Letters 95, (2009) 183104

7. R. Lifshitz, M. C. Cross, Reviews of Nonlinear Dynamics and Complexity (Wiley, Weinheim, 2008) 52

8. H. W. Ch. Postma, I. Kozinsky, A. Husain, M. L. Roukes, Applied Physics Letters 86, (2005) 223105

9. M. Sato, A. J. Sievers, Low Temperature Physics 34, (2008) 543-548

10. Y. Sarov, T. Ivanov, A. Frank, and I. W. Rangelow, Applied Physics A 92, (2008) 525-530

11. R. Lifshitz, M. C. Cross, Physical Review B (Condensed Matter and Materials Physics) 67, (2003) 134302 12. K. L. Tuer, M. F. Golnaraghi, D. Wang, Nonlinear Dynamics 5, (1994) 131-151

13. P. R. Sethna, Journal of Applied Mechanics 32, (1965) 576-582

14. A. Q. Siddiqui and M. F. Golnaraghi, Mathematical Problems in Engineering 2, (1996) 107-129

15. E. Buks, M. L. Roukes, Journal of Microelectromechanical Systems 11, (2002) 802-807

16. R. Lifshitz, M. C. Cross, Physical Review B 67, (2003) 134302

17. S. Gutschmidt, O. Gottlieb, Journal of Sound and Vibration 329, (2010) 3835-3855

18. M. R. M. Crespo Da Silva, C. C. Glynn, Journal of Structural Mechanics 6, (1978) 437-448 